\title{
“Criança não pode esperar": a busca de serviço de urgência e emergência por mães e suas crianças em condições não urgentes
}

\author{
"Children cannot wait": why mothers seek urgency and \\ emergency care services for their children in non-urgent situations
}

Rose Meire Silva Rati ${ }^{1}$

Lúcia Maria Horta de Figueiredo Goulart ${ }^{2}$

Cristina Gonçalves Alvim ${ }^{2}$

Joaquim Antônio César Mota ${ }^{2}$

${ }^{1}$ Fundação Hospitalar do Estado de Minas Gerais. Alameda Álvaro Celso 100, Santa Efigênia. 30.150-260 Belo Horizonte MG Brasil. rose.rati@gmail.com

${ }^{2}$ Departamento de Pediatria, Faculdade de Medicina, Universidade Federal de Minas Gerais.
Abstract The scope of this article is to seek to understand the reasons why mothers seek urgency and emergency attention for children in nonurgent situations in a public hospital of the Unified Health System in Belo Horizonte in the state of Minas Gerais. It is a study involving a qualitative approach with semi-structured interviews as the data collection tool. Twenty-seven mothers of children with complaints classified as minor and non-urgent were interviewed. Fever and respiratory problems were found to be the main complaints. Even when the situation is non-urgent, mothers take their children to the emergency service because of the swift resolution, quality and specific pediatric medical care advantages, ease of access, previous experiences and recommendations from others, among other reasons. The statements revealed the mismatch between these mothers' feelings and the rationale of the organization of the service. This work raises questions that can provide input for reflection on the organization of the services centered on the users, who are in the final analysis the subject and scope of the caregiving process.

Key words Emergency medical services, Demands on the health services, Child health services, Reception
Resumo O objetivo deste artigo é compreender os motivos para a busca do atendimento de urgência e emergência pelas mães, para crianças em condições não urgentes, em hospital público em Belo Horizonte. Trata-se de pesquisa qualitativa com a utilização de entrevista semiestruturada como instrumento de coleta de dados. Foram entrevistadas 27 mães de crianças classificadas como pouco ou não urgentes. Foi realizada análise de conteúdo. Febre e problemas respiratórios foram as principais queixas. Mesmo não se tratando de urgência, as mães procuram o serviço pela sua resolutividade, qualidade, especificidade para atendimento de crianças, facilidade de acesso, experiências, recomendações de terceiros, entre outros. Os depoimentos evidenciaram o descompasso entre o sentir dessas mães e a racionalidade da organização do serviço. Este trabalho aponta questões que poderão servir de subsídios para reflexão sobre a organização dos serviços centrada no usuário, sujeito e fim do processo assistencial.

Palavras-chave Serviços Médicos de Emergência, Demanda aos serviços de saúde, Serviços de Saúde da Criança, Acolhimento 


\section{Introdução}

É amplamente reconhecido que a procura por serviços de urgência e emergência para casos que poderiam ser resolvidos em serviços de menor densidade tecnológica, ocorre independente do nível de desenvolvimento econômico e social dos países ${ }^{1-4}$.

Estudos realizados na década de 1990, no Rio de Janeiro e em Recife, evidenciaram que mais de $50 \%$ dos casos atendidos em unidades pediátricas de urgência eram provenientes de demanda espontânea, percentuais reproduzidos nos últimos anos. Resultados mais recentes encontrados em prontos socorros de hospitais em Recife revelaram que apenas $15,2 \%$ dos casos que motivaram a demanda infantil eram compatíveis com o potencial tecnológico da unidade. O principal motivo apresentado para a busca desses serviços foi a preferência dos responsáveis pelas unidades de maior densidade tecnológica ${ }^{4}$. Em Maceió, pesquisa em Unidade de Emergência apontou que apenas $16,8 \%$ das crianças e adolescentes atendidos em 1998, 2001 e 2004 apresentavam agravos compatíveis com o potencial tecnológico oferecido pelo serviço ${ }^{5}$.

Em Belo Horizonte, a utilização das unidades de urgência pelo usuário é também realizada por demandas que não se enquadram necessariamente nesta definição ${ }^{6}$. O que se observa é que, apesar da progressiva expansão da rede básica e da implantação do Programa Saúde da Família, a demanda pelos serviços de urgência e emergência não vem se modificando. Constata-se divergência no modo de pensar e tratar a urgência pelos gestores, profissionais de saúde e usuários desses serviços. Apesar da disponibilidade de portas de entrada no sistema de saúde hierarquicamente estabelecidas pelos gestores e técnicos, verifica-se a busca da população pelos serviços de urgência, sem que fique clara a compreensão sobre os motivos dessa procura.

A atitude dos usuários ao buscarem hospitais, pronto socorros e pronto atendimentos, como opção inicial para atendimentos não considerados de urgência, vem sobrecarregando esses serviços e profissionais de saúde e preocupando os gestores do sistema.

Nos hospitais pediátricos, a situação é ainda mais preocupante, pois a grande demanda por atendimento merece cuidados especiais, visto que a triagem em crianças mais jovens é particularmente difícil?.

Com o objetivo de organizar a demanda e estabelecer prioridades para o atendimento nes- ses serviços, várias estratégias têm sido propostas. Uma é o acolhimento com classificação de risco. A classificação de risco permite a priorização do atendimento aos pacientes com doenças graves ou com instabilidade de órgãos/sistemas, otimizando recursos, melhorando a qualidade da assistência, orientando o usuário para a resposta mais adequada à sua demanda naquele momento, não constituindo um instrumento de diagnóstico clínico ${ }^{8}$.

Em Belo Horizonte, no sentido de organizar $\mathrm{o}$ atendimento, um grande hospital pediátrico pertencente à rede pública, implantou, em agosto de 2007, o acolhimento com classificação de risco no ambulatório de urgência e emergência, priorizando as crianças em condições consideradas de maior risco. De acordo com o perfil institucional e da clientela, a equipe desse hospital adotou um sistema semelhante ao sugerido pelo Protocolo de Manchester'. No "Protocolo de Manchester - Triagem de Prioridades na Urgência", a situação clínica do paciente é classificada em cinco níveis e identificada por cores: Emergência (vermelho), Muito Urgente (laranja), Urgente (amarelo), Pouco Urgente (verde), Não Urgente (azul). Cada criança que é levada ao serviço é acolhida e avaliada por profissionais de enfermagem, conforme a classificação proposta. De acordo com essa classificação, o atendimento médico pode ser imediato ou demorar alguns minutos ou horas. Assim, o responsável pela criança é orientado quanto ao possível tempo de espera para o atendimento desse profissional.

Estudo realizado antes da implantação do acolhimento com classificação de risco nesse mesmo ambulatório demonstrou que, apesar da vocação do serviço em atender casos de urgência e emergência, no ano de 2004 quase a metade dos atendimentos não se enquadrou nessas categorias $^{6}$. No ano subsequente à implantação do acolhimento com classificação de risco, verificou-se que, dos 64.749 atendimentos realizados naquele ambulatório no período de setembro de 2007 a setembro de 2008, 56,9\% foram classificados como pouco urgentes e não urgentes, caracterizando procura inadequada pelo serviço por pacientes em situações que poderiam ter sido resolvidas no âmbito da atenção primária ${ }^{10}$.

Os dados encontrados antes e após a implantação do acolhimento com classificação de risco demonstram que os casos de crianças que não são considerados como urgência e emergência pela instituição continuam representando a maior demanda do atendimento, sugerindo que essa estratégia, por si só, não é suficiente para ade- 
quar a demanda à vocação do serviço. Neste contexto, é de fundamental importância conhecer os motivos que levam as famílias a buscar os serviços de urgência/emergência.

A literatura sobre o comportamento das famílias na busca pelo atendimento na urgência/ emergência ainda é escassa. A ampla ocorrência desse comportamento indica a necessidade de melhor compreendê-lo, sobretudo porque o aclaramento desse processo aos profissionais de saúde pode melhorar a sua colaboração com os pais e possibilitar melhor orientação quanto ao manejo da criança doente.

Este trabalho teve como principal objetivo compreender os motivos para a busca por atendimento de urgência e emergência pelas mães, para crianças em condições não urgentes.

\section{Método}

Trata-se de uma pesquisa qualitativa, uma vez que se pretende compreender experiências vividas por mães de crianças supostamente doentes, buscando-se aspectos da realidade que não podem ser quantificados pela sua natureza única e singular: significados, motivações, sentimentos, valores, expectativas, crenças e atitudes ${ }^{11}$.

Os sujeitos deste estudo foram mães que procuraram espontaneamente o serviço de urgência e emergência de um hospital pediátrico de referência da rede pública em Belo Horizonte, visto que as mesmas são consideradas informantes privilegiadas sobre a saúde da criança e representam a maioria dos acompanhantes que buscam o serviço. Foram critérios de inclusão: ser mãe de criança em situação classificada como pouco urgente ou não urgente (cor verde ou azul, segundo o protocolo de Manchester adaptado) e residir em Belo Horizonte. As mães foram captadas na sala de espera, após avaliação da sua criança e classificação de risco pela enfermagem, enquanto aguardavam o atendimento médico.

Utilizou-se, como principal instrumento, a entrevista semiestruturada, com base em roteiro elaborado a partir da revisão da literatura afim e do estudo exploratório. Os principais temas abordados tinham como eixo a percepção da doença da criança e a busca pelo serviço. As entrevistas com as mães foram realizadas individualmente, em local apropriado no ambulatório disponibilizado para essa finalidade, com duração entre 20 e 60 minutos. Todas as entrevistas foram gravadas, com autorização da informante. Para garantir a confidencialidade das infor- mações, as mães foram identificadas com nome fictício. Logo após o término de cada entrevista, a mesma foi transcrita e digitada pela própria entrevistadora, possibilitando desta maneira melhor apreensão do conteúdo gravado.

O critério de saturação foi utilizado para a constituição da amostra. Assim, quando as informações obtidas em novas entrevistas tornaram-se repetitivas, já não mais contribuindo significativamente para a compreensão da realidade investigada e reflexão teórica nela fundamentada, considerou-se o momento da interrupção da coleta de dados ${ }^{12}$.

Foi utilizada a técnica de análise do conteúdo ${ }^{13}$. A primeira etapa, de pré-análise, consistiu na reunião de todo o material empírico, leitura flutuante do mesmo e exploração exaustiva do conteúdo de cada entrevista. Na segunda etapa, exploração do material, procedeu-se à operação de codificação, isto é, a partir dos dados brutos foi feito recorte das unidades de registro significativas por temáticas, visando alcançar o núcleo de compreensão do texto. Assim, os dados foram agrupados por temas e, num processo classificatório, foram identificadas as principais categorias: a doença da criança; o hospital de urgência e a emergência como local escolhido; urgência e emergência na perspectiva das mães. Na terceira etapa, foi realizada a análise final, com o tratamento e a interpretação dos resultados obtidos.

A triangulação entre os pesquisadores foi realizada durante todo o processo de coleta de dados e análise. A utilização da observação como instrumento coadjuvante possibilitou também a triangulação de métodos, aumentando a confiabilidade do estudo ${ }^{11}$.

A coleta de dados foi realizada no período de junho a setembro de 2008, após a aprovação do estudo pelo Comitê de Ética das duas instituições envolvidas - Universidade Federal de Minas Gerais/UFMG e Fundação Hospitalar do Estado de Minas Gerais/FHEMIG.

\section{Resultados e discussão}

Foram realizadas 27 entrevistas com mães de crianças classificadas como em condição pouco urgente (26) e não urgente (1). O perfil sociodemográfico das mães é mostrado na Tabela 1.

A idade das crianças variou entre cinco meses e 10 anos, sendo que a maioria encontrava-se na faixa etária entre um e quatro anos. Mais da metade era do sexo feminino. 
Tabela 1. Características das mães entrevistadas $(\mathrm{n}=27)$.

\begin{tabular}{|c|c|c|}
\hline & $\mathbf{n}$ & $\%$ \\
\hline \multicolumn{3}{|l|}{ Idade (anos) } \\
\hline$<20$ & 1 & 3,7 \\
\hline$\geq 20$ e $<30$ & 15 & 55,6 \\
\hline$\geq 30$ & 11 & 40,7 \\
\hline \multicolumn{3}{|l|}{ Número de filhos } \\
\hline 1 & 18 & 66,7 \\
\hline 2 ou mais & 9 & 33,3 \\
\hline \multicolumn{3}{|l|}{ Escolaridade } \\
\hline Médio completo & 11 & 40,8 \\
\hline Fundamental completo & 10 & 37,0 \\
\hline Fundamental incompleto & 6 & 22,2 \\
\hline \multicolumn{3}{|l|}{ Vive com o pai da criança } \\
\hline Sim & 19 & 70,4 \\
\hline Não & 8 & 29,6 \\
\hline \multicolumn{3}{|c|}{ Renda familiar(SM - Salário Mínimo) } \\
\hline$<2$ & 14 & 51,9 \\
\hline$\geq 2 \mathrm{e}<3$ & 10 & 37,0 \\
\hline$\geq 3$ & 3 & 11,1 \\
\hline \multicolumn{3}{|l|}{ Trabalha fora do lar } \\
\hline Sim & 14 & 51,9 \\
\hline Não & 13 & 48,1 \\
\hline \multicolumn{3}{|l|}{ Cadastro no PSF } \\
\hline Sim & 4 & 14,8 \\
\hline Não & 23 & 85,2 \\
\hline \multicolumn{3}{|c|}{$\begin{array}{l}\text { Tipo de transporte usado para chegar } \\
\text { ao hospital }\end{array}$} \\
\hline Coletivo & 24 & 88,9 \\
\hline Táxi & 1 & 3,7 \\
\hline Carro particular & 1 & 3,7 \\
\hline A pé & 1 & 3,7 \\
\hline
\end{tabular}

\section{A doença da criança}

Na percepção das mães, a doença da criança foi expressa por mudança no seu modo habitual de ser:

"Ficou tristinha, cansada, choramingando. Tristeza, desânimo, cansaço para respirar”.

As mães revelaram possuir um limite de segurança para cuidar dos filhos. Quando percebem que atingiram esse limite, elas buscam o serviço de saúde, pois precisam dos profissionais de saúde para ajudá-las nos cuidados necessários. Algumas mães informaram ter tentado alguns cuidados em casa, antes de trazer as crianças ao hospital.

"Dei dipirona, mas como a febre continuou... eu resolvi trazer ele aqui".

As queixas que motivaram a procura pelo serviço estão apresentadas na Tabela 2. Mais da
Tabela 2. Queixas que motivaram a procura pelo hospital de urgência/emergência.

\begin{tabular}{lrc}
\hline \multicolumn{1}{c}{ Queixas $^{*}$} & n & \% \\
\hline Febre & 14 & 51,8 \\
Tosse e/ ou dificuldade respiratória & 9 & 33,3 \\
Dor & 7 & 25,9 \\
Vômito & 6 & 22,2 \\
Gripe & 3 & 11,1 \\
Outros & 6 & 22,2 \\
\hline${ }^{*}$ Os motivos de procura não foram excludentes & &
\end{tabular}

* Os motivos de procura não foram excludentes

metade das mães citou a febre e a terça parte se referiu às manifestações respiratórias como tosse e/ou dificuldade para respirar.

Evidenciou-se que a presença de febre foi importante fator mobilizador para procura pelo ambulatório de urgência/emergência. Outros estudos sobre a demanda nesses serviços apontam para resultados semelhantes ${ }^{14,15}$.

Embora a febre nem sempre seja considerada pelos profissionais de saúde como sinal de gravidade e tecnicamente por si só não justifique a procura do serviço de urgência ${ }^{16}$, para as mães entrevistadas foi motivo de preocupação e ansiedade.

"A febre é sinal de infecção, alguma coisa errada, o organismo está dando um alerta, né? A gente tem de vigiar, né? Por isso é que quando ela aparece com febre, eu corro pro hospital".

Em 1980, Schmitt cunhou o termo "fever phobia" referindo-se ao sentimento de medo dos pais em relação à febre em suas crianças ${ }^{17}$. Alguns estudos referem-se aos sentimentos e comportamentos dos pais diante dessa situação tão frequente na infância, enfatizando especialmente o medo de consequências como convulsões e delírios ${ }^{18,19}$. Nesta pesquisa, o "pavor de febre" aparece em alguns depoimentos:

"Eu tenho pavor de febre! Pode dar convulsão. Se deu febre, nossa!!! Aí eu já preocupo... é uma preocupação a mais. Febre para mim é pavor, trauma...."

\section{O hospital de urgência e emergência como local escolhido}

Estudos mostram que os usuários escolhem os serviços de saúde não apenas pela localização geográfica, mas também pela resolutividade, qualidade do serviço, garantia e agilidade no acesso, por experiências positivas no passado, pelo acolhimento prestado, bem como pela avaliação 
que o mesmo faz do seu estado de saúde 20 -23. Fatores culturais interferem nessa escolha e a percepção de situações "simples” e "graves" resulta em demanda espontânea.

Neste trabalho evidenciou-se que são vários os motivos para a escolha do serviço de urgência e emergência: resolutividade, qualidade, especificidade para atendimento de crianças, facilidade de acesso, experiências e recomendações:

"Porque já consulta, já passa os remédios, tem médico e tudo fica resolvido pra ir embora".

"É como eu te falei, aqui é mais desenvolvido, eu acho que o atendimento é melhor. A gente fica mais confiante, né?".

"Aqui é hospital só infantil. Aqui tem pediatra".

“Trago aqui. É porque é mais fácil. Apesar que demora muito, mas é central. $\mathrm{O}$ acesso é mais fácil."

"Dou preferência aqui, desde que eu fui bem atendida da primeira vez".

"Minha vizinha falou que aqui é muito bom".

O serviço foi percebido como o local que congrega consultas a especialistas, investigação e tratamento de doenças. O usuário busca o atendimento na urgência/emergência por acreditar que as ações de saúde, que lhe são oferecidas, são imediatas e resolutivas. Estudo realizado no Rio Grande do Sul constatou que as pessoas procuravam os serviços de pronto atendimento para a solução de seus problemas de saúde, independentemente da sua gravidade ${ }^{21}$. Observa-se que o usuário quer ser atendido e para tal ele busca os caminhos que são do seu conhecimento e os serviços que dão acesso ao atendimento de sua demanda.

As mães expressaram sua opinião de que o atendimento da criança não deve ser realizado junto com o atendimento de adulto, justificando sua preferência pelo hospital exclusivamente infantil. Elas demonstraram medo de que a criança presencie cenas trágicas ou até mesmo seja contaminada pela doença dos adultos.

"No pronto socorro você é atendido, mas tem aquele tanto de gente doente, gritando, com sangue. Isto pode dar até um trauma nela. Criança não pode ver estas coisas".

O fato de o atendimento médico ser realizado por pediatra também foi valorizado pelas mães. Nos seus depoimentos as mães expressaram maior confiança no desempenho desse profissional em relação ao do generalista. A valorização do especialista foi também um fator importante na determinação da demanda dos usuários do SUS a prontos-socorros e hospitais em
Juiz de Fora $(\mathrm{MG})^{23}$. O papel do pediatra na Estratégia de Saúde da Família diante dos múltiplos desafios para o desenvolvimento de prática que responda às necessidades individuais e coletivas, pautada pelos princípios do SUS, ainda é tema de debates e controvérsias ${ }^{24}$. Enquanto que, em unidades de atendimento de urgência e emergência, a presença desse profissional é quase obrigatória, sendo ele, na maioria das vezes, o primeiro a prestar atendimento à criança doente, nas Unidades Básicas de Saúde, esse primeiro atendimento é realizado pelo médico generalista.

A facilidade de acesso ao hospital em questão foi evidenciada como motivo de sua escolha em diversos depoimentos. O hospital está situado em região central e hospitalar da cidade, que é atendida por várias linhas de ônibus. Conforme informação dada pelas mães, o tempo gasto para se chegar naquele local variou de 15 a 60 minutos. Quase 90\% das mães entrevistadas informaram ter utilizado transporte coletivo.

Experiências positivas anteriores com o atendimento nesse serviço, próprias ou de pessoas próximas, foram também decisivas para que as mães optassem pelo mesmo local. O Hospital onde se realizou a pesquisa é referência em pediatria de Belo Horizonte e região metropolitana e é muito conhecido pela população usuária do Sistema Único de Saúde (SUS). Esta instituição funciona no mesmo local desde 1982 e nunca teve interrupção no atendimento. Parentes, amigos, vizinhos indicam o hospital e a clientela demonstra certo vínculo com essa instituição:

"Aqui é o lugar certo, aqui a mãe fica tranquila".

Estudo sobre a resolutividade dos problemas de saúde na perspectiva dos usuários de uma Unidade Básica de Saúde de Porto Alegre (RS) apontou que a procura por determinado serviço de saúde não ocorre apenas pelo desejo ou pela esperança de atendimento; fundamenta-se em avaliação de experiências vividas que determinam qual serviço deverá ser acessado ${ }^{25}$.

Neste trabalho, a busca pelo serviço de urgência/emergência em situações que poderiam ser resolvidas no âmbito da atenção primária emerge como ponto de questionamento e reflexão sobre a organização da assistência à saúde em Belo Horizonte. Sabe-se que a cidade conta com 147 centros de saúde distribuídos em nove regionais, com cobertura de $77 \%$ da população pelo Programa de Saúde da Família. As áreas mais carentes estão todas cobertas, sendo que o menor índice de cobertura pelo Programa de Saúde da Família refere-se à população de melhor po- 
der aquisitivo. Belo Horizonte é considerada modelo de referência para organização da rede pública de atenção primária à saúde no Brasil. Uma pesquisa realizada em 2010 revelou que $75,3 \%$ da população estava satisfeita/muito satisfeita com o trabalho da equipe e que 86,6 estava satisfeita/muito satisfeita com os serviços ofertados pelo Centro de Saúde de referência. Em relação à satisfação com o acesso ao sistema de saúde, a proporção de satisfeitos/muito satisfeitos cai para $60,7 \%{ }^{26}$.

Interessante notar que apesar da ampla cobertura da atenção primária e da alta proporção de satisfação com esses serviços, evidenciada naquela pesquisa, a procura do serviço de urgência/emergência por problemas de saúde que poderiam ser resolvidos na Unidade Básica de Saúde continua sendo um desafio para os gestores e para os profissionais que lidam cotidianamente com essa demanda.

Neste estudo, verificou-se que das 27 mães entrevistadas apenas quatro informaram que estavam cadastradas na Unidade Básica de Saúde (UBS) de referência para o seu domicílio. Entretanto, com exceção de duas mães que desconheciam a UBS responsável pela área de sua moradia, todas as demais revelaram conhecer esse serviço e traziam consigo no momento da entrevista a Caderneta de Saúde da Criança ou o Cartão da Criança, que é o instrumento de registro de informações de saúde na atenção primária. Nos seus depoimentos, as mães apontaram motivos pelos quais não optaram pela UBS - porta de entrada natural no processo de assistência à saúde - para atendimento dos seus filhos naquela situação:

"Eu vou lá (na UBS) mais para controle, vacina..."

"O que eu faço aqui, eu não faço lá no Posto: um RX na hora, um exame de urina. Exame lá demora uma semana, 10 dias..."

"Uma vez eu fui lá no Posto com ela e não tinha nem termômetro. Imagina! Dentro de um Posto de Saúde! Lá é muito carente, não tem estrutura".

Então se passa mal de noite e final de semana é só aqui mesmo. Quando dá eu levo lá em dia de semana"

"Ah... porque lá no Posto demora muito pra atender..."

"Aqui você consulta com médico. Lá no Posto você espera duas, três horas e ainda é para ser atendido por uma enfermeira

"Aí eu perdi a confiança no Posto, porque lá só olha a garganta e o ouvido e mais nada. Tudo é virose!"
"No Posto, muitas vezes, o médico é um clínico geral, ele não entende de criança, não é formado para atender criança".

O Quadro 1 sintetiza os motivos pelos quais as mães não optaram pelo serviço de atenção primária para o atendimento da sua criança.

Estudo realizado em Juiz de Fora (MG) apontou que a disponibilidade de recursos na unidade de saúde - incluindo desde medicamentos básicos até recursos tecnologicamente mais sofisticados - também é componente significativo da imagem de qualidade que o usuário tem sobre $\mathrm{o}$ cuidado de saúde a ser recebido. Nessa pesquisa, o discurso dos usuários participantes elucidou o quanto a imagem sobre os serviços de saúde é afetada em função de sua maior ou menor disponibilidade de recursos ${ }^{23}$.

Em Belo Horizonte, pesquisa sobre a procura de atendimento de urgência nas Unidades de Pronto Atendimento (UPA) mostrou que a garantia de atendimento, a disponibilidade tecnológica em termos de equipamento, $o$ atendimento médico, a rapidez e a agilidade para resolver o problema de saúde foram os principais determinantes dessa procura. A falta de resolutividade das UBS foi recorrente na fala dos entrevistados participantes dessa pesquisa ${ }^{27}$.

Os resultados de avaliação do Programa Saúde da Família (PSF) em dez grandes centros urbanos do País evidenciaram que, na maior parte dos municípios estudados, as Unidades de Saúde da Família (USF) ainda não se tornaram a porta de entrada de um sistema integrado de atenção à saúde. O estudo sugere que a baixa proporção da procura da USF, frente a episódio de doença, pode estar relacionada às barreiras de acesso de-

Quadro 1. Motivos alegados pelas mães para a não opção pela UBS.

\begin{tabular}{|l|}
\hline $\begin{array}{l}\text {. a UBS é apropriada para o controle de crianças } \\
\text { sadias }\end{array}$ \\
\hline $\begin{array}{l}\text {. a UBS não oferece exames complementares "na } \\
\text { hora” }\end{array}$ \\
\hline . a infraestrutura da UBS é precária \\
\hline . o horário de atendimento é limitado \\
\hline . há demora no atendimento \\
\hline . falta confiança no atendimento do médico \\
\hline . falta compreensão do papel do enfermeiro \\
\hline $\begin{array}{l}\text {. O atendimento da criança é realizado por } \\
\text { generalista e não por pediatra }\end{array}$ \\
\hline
\end{tabular}


correntes de horários de funcionamento, adscrição ao local de moradia (e não de trabalho), às dificuldades em garantir simultaneamente atendimento à demanda espontânea e aos grupos não prioritários, e à implantação recente do serviço. Como conclusão, os resultados indicaram ser necessário repensar horários de funcionamento das Unidades e estabelecer fluxos de informação e referência entre os serviços de urgência e de pronto atendimento e as $\mathrm{USF}^{28}$.

Já na cidade de Recife, entrevista com 939 usuários de Unidades de Saúde da Família, mostrou a vinculação e a alta procura por aqueles serviços em situação de problemas de saúde (86,2\%). Contudo, o estudo revelou a insatisfação com os tempos de espera para o atendimento $(54,7 \%)$, para agendar consulta especializada $(47,5 \%)$ e na própria unidade $(45,3 \%)$ e ainda para receber os resultados dos exames realizados (63\%), evidenciando as dificuldades relacionadas à organização daqueles serviços ${ }^{29}$.

Apesar dos significativos avanços no processo de descentralização, a utilização da rede de atenção primária como porta de entrada ainda é marcada por contradições que se evidenciam no confronto entre as demandas individuais e os serviços oferecidos, o grau de resolubilidade, as relações interpessoais e a densidade tecnológica, entre outros fatores. Assim, os ambulatórios de pronto-atendimento e serviços de urgência/emergência, embora superlotados e impessoais, ainda se constituem como importantes portas de entradas no sistema de saúde, representando a possibilidade de realização imediata de consultas, procedimentos, exames complementares, aquisição de medicamentos e internação, se necessário.

\section{Urgência e emergência na percepção das mães: "criança não pode esperar"}

Jacquemot ${ }^{15}$, pesquisando as perspectivas de usuários e profissionais, encontrou que as pessoas geralmente não estabelecem diferenças, em termos de graus de gravidade, entre urgência e emergência. Falavam em urgência e quando queriam significar que era grave diziam "é muito urgente", "é uma urgência mesmo". A palavra emergência foi pouco presente nas falas dos usuários, sendo as palavras "urgência" e "urgente" mais familiares.

Neste trabalho, as informantes mostraram dificuldades em conceituar e diferenciar urgência e emergência, o que seria esperado, pois são conceitos técnicos cuja diferenciação, para leigos, pode não ser clara. Entretanto, algumas mães demonstraram ter uma concepção de urgência e emergência próxima ou semelhante ao conceito adotado na organização do serviço, possivelmente por apropriação do discurso técnico científico ressignificado por suas vivências.

"Urgência tá grave, né? Emergência muito mais grave, tem de ser atendido primeiro".

Nas entrevistas, as mães reconheciam que existiam casos de maior gravidade do que o de seu filho, porém consideravam que, por ser sua criança, tratava-se sempre de emergência.

"E no caso de seu filho, é emergência. Para a mãe é isso, tudo é emergência”.

A urgência, em algumas situações, se referia à necessidade de se retomar a rotina familiar e se livrar de um transtorno ou de um incômodo.

"O problema dela é de urgência sim. O problema dela é grave porque ela não deixa ninguém sossegado. Ela não deixou ninguém dormir esta noite..."

"Não é urgência, mas mesmo assim eu trouxe aqui. Precisa melhorar a tosse com urgência, ela tá muito incomodada”.

Mesmo a criança recebendo classificação como pouco urgente, cor verde ou classificação não urgente, cor azul, as mães preferiam esperar o atendimento com medo de que a situação se agravasse:

"No caso da minha filha não é grave, mas não pode esperar. Pra mim tudo é grave do jeito que ela tá. E se vira uma pneumonia?”

Fica claro, portanto, que a finalidade para o usuário da procura por serviço de urgência/emergência é diferente daquela identificada pelo profissional da instituição, definida no modelo biológico e na organização do trabalho. O usuário busca o serviço para solução de suas necessidades que, graves ou não, naquele momento, lhe trazem angústia e desconforto.

Embora haja definições técnicas para urgência e emergência, o pressuposto é de que a população usuária possui definição própria que a leva a procurar por esse serviço ${ }^{30}$. Do ponto de vista da gestão, procurar o serviço de emergência em situações que não se caracterizam como tal é um problema. Entretanto, para o sujeito "doente" pode ser "solução", pela possibilidade de alívio mais rápido para seu sofrimento.

Constata-se que há uma distância entre o que é urgência para a mãe e o que é para o profissional de saúde. Para as mães, a urgência está baseada em critérios subjetivos, culturais e sociais. Para o profissional de saúde, os critérios são técnicocientíficos. Essa distância entre o significado de urgência para o usuário e para os profissionais 
de saúde tem sido também constatada em outros países. Estudo americano realizado na década de 90 mostrou a baixa concordância (26\%) entre o julgamento dos pais sobre a gravidade das doenças de suas crianças e o julgamento dos médicos ${ }^{31}$. Já na Austrália, estudo qualitativo sobre as atitudes e as percepções dos pais a respeito da emergência pediátrica mostrou divergências com as dos profissionais de saúde, resultando em insatisfações em ambos os grupos e potencializando problemas na utilização dos serviços de saúde $^{32}$. No Canadá, Truman e Reutter ${ }^{33}$, estudando o comportamento de 114 pais que levaram suas crianças em condições não urgentes ao serviço de urgência/emergência de um grande hospital, demonstraram que 58\% não havia tentado contato com o médico de família e $82 \%$ revelou insegurança diante dos sintomas das suas crianças ou superestimou a sua gravidade.

Jacquemot ${ }^{15}$ enfatiza que o conhecimento do contexto é fundamental para entender as urgências dos usuários. Pode ser que não seja tanto a doença em si que gere uma demanda de intervenção urgente, mas o contexto geral no qual esta se manifesta e perturba. Nesse sentido, a urgência torna-se construção coletiva da qual participam o doente, e também sua família e até os vizinhos. Para esclarecer melhor o aspecto contextual, essa autora explica que os profissionais de saúde possuem concepção especializada, "singularizante" e "autonomizada" da urgência. Os usuários, por sua vez, têm concepção "globalizante", que contrasta com a concepção biomédica. As urgências vividas por leigos são configurações ameaçadoras, caracterizadas não somente pelo problema de saúde, mas também associada com outros riscos que colocam a vida em perigo. Nesse sentido, suas urgências indicam não só suas necessidades, mas, sobretudo, os transtornos e sofrimentos vivenciados - é o que "não pode esperar":

\section{Colaboradores}

RMS Rati, LMHF Goulart, CG Alvim e JAC Mota participaram igualmente de todas as etapas de elaboração do artigo.
"Doença não aguenta esperar. Ainda mais criança. Eu não espero. Adulto parece que aguenta mais, é mais forte. A criança fica quase sem respirar, não pode dar qualquer remédio. Criança não pode esperar."

\section{Conclusão}

Este estudo apontou caminhos para a compreensão da procura do serviço de urgência e emergência pelas mães, para atendimento de suas crianças em condições não urgentes. A percepção singularizada da doença da criança e o medo da piora aliados à certeza de um atendimento resolutivo foram fatores decisivos para essa procura.

Evidenciou-se que a concepção de urgência/ emergência para as mães é bem diferente daquela adotada pela instituição. Há um descompasso entre o sentir das mães e a racionalidade do serviço, o que gera conflitos, insatisfação e uma demanda espontânea que acaba por inverter a hierarquização na atenção à saúde.

Por outro lado, ao buscar compreender os motivos para a busca por atendimento na urgência/emergência surgem questionamentos sobre a acessibilidade e a adesão aos serviços de atenção primária onde, de acordo com a lógica da hierarquização e regionalização, essas crianças em situação não urgente poderiam ter recebido atendimento com resolutividade.

$\mathrm{O}$ estudo possibilitou que as mães expressassem suas dificuldades com os serviços de atenção primária, o que ampliou a compreensão sobre a opção pelo de urgência/emergência.

Finalmente, ao apontar contradições no fluxo da demanda numa cidade considerada modelo de organização da atenção à saúde, esta pesquisa fornece subsídios para uma reflexão que busque a centralidade do usuário na construção de um SUS mais acessível e resolutivo. 


\section{Referências}

1. Pope D, Fernandes CMB, Bouthillete F, Etherington J. Frequent users of the emergency department: a program to improve care and reduce visits. CMAJ 2000; 162(7):1017-1020.

2. Ryan M, Spicer, M, Hyett C, Barnett, P. Non-urgent presentations to a paediatric emergency department: Parental behaviours, expectations and outcomes. EMA 2005; (17):457-462.

3. Berry A, Brousseau D, Brotanek J M, Tomany-Korman S, Flores G. Why do parents bring children to the Emergency Department for Nonurgent conditions? A qualitative study. Ambul Pediatr 2008; 8(6):360-367.

4. Kovacs MH, Feliciano KVO, Sarinho SW, Veras AACA. Acessibilidade às ações básicas entre crianças atendidas em serviços de pronto-socorro. Jornal de Pediatria 2005; 81(3):251-258.

5. Simons DA, Monlléo IL, Simons AS, Júnior JLA. Adequação da demanda de crianças e adolescentes atendidos na Unidade de Emergência em Maceió, Alagoas, Brasil. Rev. Bras. Saúde Matern. Infant. 2010; 10(1):59-67.

6. Melo EMC, Assunção AA, Ferreira RA. O trabalho dos pediatras em um serviço público de urgência: fatores intervenientes no atendimento. Cad Saude Publica 2007; 23(12):3000-3010.

7. van Veen M, Steyerberg EW, Ruige M, Meurs AHJ, Roukema J, Lei J V, Moll H A Moll. Manchester triage system in paediatric emergency care: prospective observational study. BMJ 2008; 337:a1501.

8. Brasil. Ministério da saúde (MS). Secretaria de assistência à saúde. Núcleo técnico da política nacional de humanização. Acolhimento com classificação de risco. Brasília: MS; 2004.

9. Manchester Triage Group. Emergency triage Group. 2a Edition. Oxford: BMJ publishing group; 2006.

10. Fundação Hospitalar do Estado de Minas Gerais. Hospital Infantil João Paulo II. Boletim Estatístico. Belo Horizonte: Fundação Hospitalar do Estado de Minas Gerais; 2007.

11. Minayo MCS. Análise qualitativa: teoria, passos e fidedignidade. Cien Saude Colet 2012; 17(3):621-626.

12. Minayo MCS. $O$ desafio do conhecimento: pesquisa qualitativa em saúde. $8^{a}$ Edição. São Paulo, Rio de Janeiro: Hucitec, Abrasco; 2004.

13. Bardin L. Análise do conteúdo. Lisboa: Edições 70; 2006.

14. Mello DF, Ferriani MGC. Estudo exploratório de opiniões de mães sobre a saúde das crianças menores de 5 anos. Rev Latino-Am. de Enfermagem 1996; 4(2):97-100.

15. Jacquemot AC. Urgências e emergências em saúde: perspectivas de profissionais e usuários. Rio de Janeiro: Fiocruz; 2005.

16. Brasil. Ministério da Saúde (MS). Secretaria de Políticas de Saúde. AIDIP Atenção integrada às doenças prevalentes na infância: curso e capacitação - avaliar e classificar a criança de 2 meses a 5 anos de idade. 2a Edição. Brasília: MS; 2003. (Série F. Comunicação e educação em saúde).
17. Schmitt BD. Fever phobia: misconceptions of parents about Fevers. Am J Dis Child 1980; 134(2):176181.

18. Al-Nouri Luay \& Basheer Khalid. Mothers' perceptions of fever in children. J Trop Pediatrics 2006; 52(2):113-116.

19. Langer T, Pfeifer M, Sönmez A, Tarhan B, Ostermann T. Appraisal of childhood fever by German and Turkish mothers in Germany-results of pilot study. Turk J Pediatrics 2010; 52(5):471-480.

20. Ramos DD, Lima MADS. Acesso e acolhimento aos usuários em uma unidade de saúde de Porto Alegre, Rio Grande do Sul, Brasil. Cad Saude Publica 2003; 19(1):21-34.

21. Ludwig MLM, Bonilha ALL. O contexto de um serviço de emergência: com a palavra o usuário. Rev. Bras. Enfermagem 2003; 56(1):12-17.

22. Marques GQ, Lima MADS. Demandas de usuários a um serviço de pronto atendimento e seu acolhimento ao sistema de saúde. Rev Latino-Am Enfermagem 2007; 15(1):13-19.

23. Oliveira LH, Mattos, RA, Souza AIS. Cidadãos Peregrinos: os 'usuários' do SUS e os significados de sua demanda a pronto-socorros e hospitais no contexto de um processo de reorientação do modelo assistencial. Cien Saude Colet. 2009; 14(5):1929-1938.

24. Rivoredo, CRSF, Oliveira, GN, Mendes, RT. A prática pediátrica no SUS: reflexões sobre o papel dos pediatras na Estratégia de Saúde da Família. Cien Saude Colet 2011; 16(10):4221-4228.

25. Degani VC. A resolutividade dos problemas de saúde: opinião de usuários em uma Unidade Básica de Saúde [dissertação]. Porto Alegre: Universidade Federal do Rio Grande do Sul; 2002.

26. Observatório de Recursos Humanos em Saúde Faculdade de Ciências Econômicas, UFMG. Monitoramento de resultados, desempenho e satisfação dos usuários da Estratégia de Saúde da Família: um estudo em Belo Horizonte. [página na Internet] 2011. [acessado 2012 jan 11]. Disponível em: http:/ /www.rededepesquisaaps.org.br/UserFiles/File/ estudoBh.pdf

27. Rocha AFS. Determinantes da procura de atendimento de urgência pelos usuários nas unidades de Pronto Atendimento da Secretaria Municipal de Saúde de Belo Horizonte [dissertação]. Belo Horizonte: Universidade Federal de Minas Gerais; 2005.

28. Giovanella L, Escorel S, Mendonça MHM. Porta de entrada pela atenção básica? Integração do PSF à rede de serviços de saúde. Saúde em Debate 2003; 27(65):278-289.

29. Santiago RF, Mendes ACG, Miranda GMD, Duarte PO, Furtado BMASM, Souza WV. Qualidade do atendimento nas Unidades de Saúde da Família no município de Recife: a percepção do usuários. Cien Saude Colet 2013; 18(1):35-44.

30. Barros DM, Sá MC. O processo de trabalho em saúde e a produção do cuidado em uma unidade de saúde da família: limites ao acolhimento e reflexos no serviço de emergência. Cien Saude Colet 2010; 15(5):2473-2482. 
31. Foldes SS, Fischer LR, Kaminsky K. What is an emergency? The judgments of two physicians. Ann Emerg Med 1994; 23(4):833-840.

32. Woolfenden S, Ritchie J, Hanson R, Nossar V. Parental use of a paediatric emergency department as an ambulatory care service. Aust N Z J Public Health 2000; 24(2):204-206.

33. Truman CD, Reutter L. Care-giving and care-seeking behaviours of parents who take their children to an emergency department for non-urgent care. Can J Public Health 2002; 93(1):41-46.

Artigo apresentado em 30/06/2012

Aprovado em 18/08/2012

Versão final apresentada em 25/08/2012 